\title{
Neurocritical Care: A Growing International Collaborative
}

\author{
Andreas H. Kramer ${ }^{1,2,3^{*}}$ (D) and Philippe Couillard ${ }^{1,2,3}$ \\ (C) 2019 Springer Science+Business Media, LLC, part of Springer Nature and Neurocritical Care Society
}

Patients with neurological conditions account for at least $10-15 \%$ of admissions to intensive care units (ICUs) [1]. In addition, many critically ill patients with sepsis or respiratory failure develop neurological complications, such as delirium, non-convulsive status epilepticus, or neuromuscular weakness, which may in turn contribute to morbidity and an increased risk of mortality [2-4]. Neurocritical care is a maturing subspecialty of critical care medicine that seeks to integrate content expertise in critical care neurology, skill and experience in general critical care management, and consistent provision of evidencebased practices for patients with brain or spinal cord injuries. The aim is to provide meticulous neuroprotection, avoidance of secondary neurological injury, prompt recognition and treatment systemic complications, and, ultimately, the best possible recovery.

A large number of observational studies have suggested that the addition of specific expertise in neurocritical care within a healthcare system is strongly associated with reductions in mortality and improved outcomes (Fig. 1) $[5,6]$. The strongest evidence supporting neurocritical care models has been for patients with spontaneous intracerebral and subarachnoid hemorrhage. More recent publications (with some exceptions), including several from outside North America or Europe, have largely reported similar results [7-18]. There is also growing evidence that higher patient volume within a center is associated with improved outcomes $[19,20]$.

\footnotetext{
*Correspondence: andreas.kramer@albertahealthservices.ca ${ }^{1}$ Department of Critical Care Medicine, Cumming School of Medicine, University of Calgary, ICU Admin - McCaig Tower, 3134 Hospital Drive NW, Calgary, AB T2N 5A1, Canada

Full list of author information is available at the end of the article
}

This comment refers to the article available at https://doi.org/10.1007/ s12028-019-00835-z.
Relevant patient-centered outcomes or quality indicators that have been highlighted in recent research extend beyond just mortality and neurological recovery, and also include aspects like good communication, realistic and accurate neuroprognostication (coupled, when appropriate, with compassionate end-of-life care), and family satisfaction [21, 22]. Neurocritical care units bring together a group of highly skilled, multidisciplinary healthcare professionals that repeatedly care for similar types of conditions, enabling them to develop expertise, refine their practices, and remain "up to date" within a defined specialty area, making them well positioned to provide exceptional care. Dedicated neurocritical care units are also an ideal environment to provide focused education and promote research.

It must, however, be recognized that outstanding neurocritical care can be provided using a variety of models (Fig. 1). What matters most is not necessarily the specific educational and professional background of healthcare providers, but rather how care is actually delivered in practice. Accordingly, a large North American multicenter study recently suggested that risk-adjusted mortality in patients with severe traumatic brain injury (TBI) was strongly associated with the presence of standardized management protocols, but not the specific designation of the unit in which care was provided [18]. One of the historical advantages of dedicated neurocritical care units has likely been that they promote adherence to standardized, evidence-based protocols, at least as a "minimum" starting point [23-25]. However, such protocols can generally be incorporated into any ICU setting. Nevertheless, the maturation of individualized, "precision," care utilizing novel neurological monitoring and guided by the unique physiologic characteristics of a particular patient is a key aspiration in the field of neurocritical care [26].

\section{Springer}




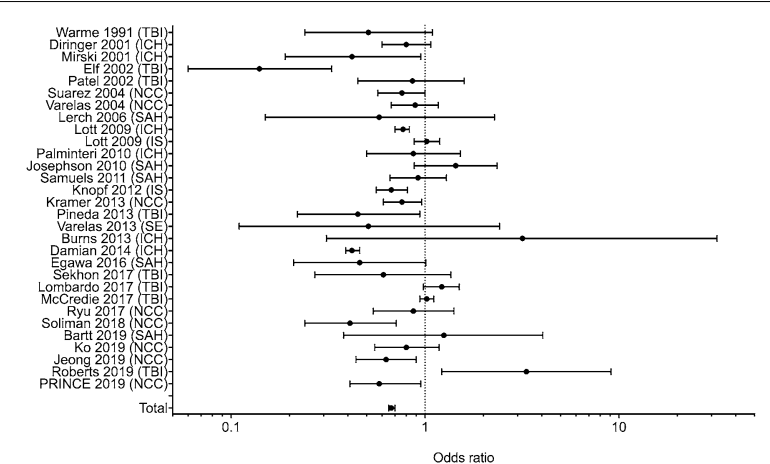

Fig. 1 Summary of observational studies assessing the association between availability of neurocritical care services and mortality (odds ratios $<1.0$ indicate lower mortality with neurocritical care). ICH intracerebral hemorrhage, IS ischemic stroke, NCC neurocritical care (multiple diagnoses), SAH subarachnoid hemorrhage, SE status epilepticus, TBI traumatic brain injury

In this edition of Neurocritical Care, as well as a previous manuscript, the authors of the Point Prevalence in Neurocritical Care (PRINCE) study have provided a contemporary snapshot of the various models used in the worldwide practice of neurocritical care $[27,28]$. The characteristics of patients admitted to participating ICUs on a single day in 2014 were observed over a period of 7 days, together with information about the environment in which care was being delivered.

Participation in the study was voluntary, such that involvement was largely restricted to centers where motivated investigators were able to input data. The study was international, but almost half of participating hospitals and more than two-thirds of patients were from the United States (USA). Thus, it is somewhat unclear to what degree the data are truly representative of worldwide practices. Because of the relatively small number of international patients, the authors have combined them (in spite of their substantial heterogeneity) to perform a comparison of patient characteristics and practices in the USA in relation to other countries.

We have highlighted several key findings that we consider to be particularly relevant:

- The PRINCE study was performed at 147 hospitals, in 31 countries, across several continents. The investigators were able to obtain high-quality data with relatively few missing elements. International collaboration will be necessary in order to address important research questions in neurocritical care. Learnings from the PRINCE study could be used as a foundation for the further development of international registries and prospective cohort studies.
- Subarachnoid hemorrhage (SAH) and intracerebral hemorrhage ( $\mathrm{ICH}$ ) are the most common diagnoses at centers participating in the PRINCE study. The neurocritical care community therefore has the opportunity to take the lead in conducting collaborative research and advancing knowledge particularly for patients with these conditions. This observation also demonstrates the importance of ensuring that healthcare professionals in our ICUs receive adequate education specifically about these conditions and are consistently very familiar with published guidelines and best practices [24, 25].

- There are many patients with brain injury that are not routinely cared for by neurocritical care specialists. The annual incidence of hospital admissions for TBI and hypoxic-ischemic brain injury (HIBI) from out-of-hospital cardiac arrest in the USA is about 85-95 and 50-60 per 100,000 population [29, 30], respectively, which dwarfs the rate of spontaneous SAH and ICH (5-20 per 100,000 per year) [31, 32]. However, patients with TBI accounted for only $6 \%$ of the PRINCE cohort (12\% if spontaneous subdural hematomas are categorized as TBI), and apart from Europe, there were almost no patients with HIBI. This is likely because patients with TBI and HIBI are commonly cared for in surgical/trauma and medi$\mathrm{cal} /$ cardiac ICUs, respectively. Given that the cause of morbidity and mortality in TBI and HIBI is most often related to the severity of brain injury, increased involvement of neurocritical care professionals in the care of these patients should be promoted, either in a primary care or in a supportive role.

- There is substantial heterogeneity in the availability of neurocritical care beds across the world. Most notably, the average number of ICU and neurocritical care beds in US hospitals was 80 (IQR 48-120) and 18 (IQR 13-24), respectively, which is more than twice that of other regions. Accordingly, there are differences in admission criteria. Specifically, patients in the USA had higher median admission Glasgow Coma Scale scores [14 (8-15) vs. 10 (5-14), $p<0.0001]$ and were less likely to require hemodynamic or respiratory monitoring. Further work is required to better define the optimal number of neurocritical care beds within a geographic area. It seems likely that there are insufficient neurocritical care resources in some regions.

- The proportion of participating hospitals that had dedicated neurocritical care units was $83 \%$ in the USA, compared with only $56 \%(p<0.0001)$ in other countries. Neurocritical care patients outside the USA are more likely to be cared for in a multi-system ICU setting. Regions without dedicated neurocritical 
care units should explore models for accessing neurocritical care expertise within their existing framework.

- There are significant international differences in nursing staffing ratios and the degree of involvement of other health professionals. Availability of dedicated pharmacists and respiratory therapists is almost universal (and generally considered to be essential), in North America, but is less consistent in other parts of the world. Nurse practitioners and physician assistants, while common in the USA, are almost nonexistent in some regions. In contrast, the availability of physiotherapists appears to be greater outside of the USA. Further research is needed to better define how best to integrate these disciplines into the management of neurocritical care patients.

- There are major cultural differences in end-of-life care practices. "Do Not Resuscitate" and "comfort care" orders preceded the vast majority of deaths occurring in North America, Australia, and New Zealand, but these practices were uncommon in Asia and the Middle East and relatively less common in Europe and Latin America. Given the importance of practices related to withdrawal of life-sustaining interventions in determining mortality rates, this observation may be an important consideration in comparative effectiveness research and in the planning of future international clinical trials.

- The findings of PRINCE corroborate previous observations from cohort studies, now involving more than 50,000 total patients, demonstrating that the presence of neurocritical care units and consultative services is associated with markedly lower mortality (Fig. 1) [5, 6]. The PRINCE study is the first truly international study to show such an association.

In summary, the PRINCE study demonstrates the feasibility of conducting international, prospective research in neurocritical care. Similar collaboration will be necessary to conduct future studies aimed at improving outcomes. Dedicated neurocritical care units, where care is provided by a collaborative team consisting of neurointensivists together with other healthcare professionals that have particular interest and experience in this area, and further supported by strong relationships with neurosurgical colleagues and other consultants, describes an ideal organizational approach to providing care for critically ill patients with brain or spinal cord injury. However, variations of this model are commonly and successfully used across the world.

\section{Author details}

${ }^{1}$ Department of Critical Care Medicine, Cumming School of Medicine, University of Calgary, ICU Admin - McCaig Tower, 3134 Hospital Drive NW, Calgary, ABT2N 5A1, Canada. ${ }^{2}$ Department of Clinical Neurosciences, Cumming School of Medicine, University of Calgary, Calgary, AB, Canada. ${ }^{3}$ Hotchkiss Brain Institute, Calgary, AB, Canada.

\section{Publisher's Note}

Springer Nature remains neutral with regard to jurisdictional claims in published maps and institutional affiliations.

Published online: 30 September 2019

\section{References}

1. Pelosi P, Ferguson ND, Frutos-Vivar F, et al. Management and outcome of mechanically ventilated neurologic patients. Crit Care Med. 2011;39:1482-92.

2. Ely EW, Shintani A, Truman B, et al. Delirium as a predictor of mortality in mechanically ventilated patients in the intensive care unit. JAMA. 2004;291:1753-62.

3. Oddo M, Carrera E, Claassen J, et al. Continuous electroencephalography in the medical intensive care unit. Crit Care Med. 2009;37:2051-6.

4. De Jonghe B, Sharshar T, Lefaucheur JP, et al. Paresis acquired in the intensive care unit: a prospective multicenter study. JAMA. 2002;288:2859-67.

5. Kramer AH, Zygun DA. Do neurocritical care units save lives? Measuring the impact of specialized ICUs. Neurocrit Care. 2011;14:329-33.

6. Kramer AH, Zygun DA. Neurocritical care: why does is make a difference? Curr Opin Crit Care. 2014;20:174-81.

7. Egawa S, Hifumi T, Kawakita K, et al. Impact of neurointensivst-managed intensive care unit implementation on patient outcomes after aneurysmal subarachnoid hemorrhage. J Crit Care. 2016;32:52-5.


ment in patients with aneurysmal subarachnoid hemorrhage after transitioning from neurosurgery led care to a neurology led multidisciplinary approach. J Vasc Interv Neurol. 2019;10:30-3.

9. Ko MA, Lee JH, Kim JG, et al. Effects of appointing a full-time neurointensivist to run a closed-type neurological intensive care unit. J Clin Neurosci. 2019;15:360-8.

10. Jeong JH, Bang J, Jeong W, et al. A dedicated neurological intensive care unit offers improved outcomes for patients with brain and spine injuries. J Intensive Care Med. 2019;34:104-8.

11. Ryu JA, Yang JH, Chung CR, et al. Impact of neurointensivist co-management on the clinical outcomes of patients admitted to a neurolosurgical intensive care unit. J Korean Med Sci. 2017;32:1024-30.

12. Lombardo S, Scalea T, Sperry J, et al. Neuro-, Trauma-, or Med/Surg-ICU: does it matter where polytrauma patients with TBI are admitted? Secondary analysis of AAST-MIST decompressive craniectomy study. J Trauma Acute Care Surg. 2017:82:489-96.

13. Roberts DJ, Leonard SD, Stein DM, et al. Can trauma surgeons keep up? A prospective cohort study comparing outcomes between patients with traumatic brain injury cared for in a trauma versus neuroscience intensive care unit. Trauma Surg Acute Care Open. 2019;4:e000229.

14. Quimby AE, Shamy MCF, Rothwell DM, et al. A novel neuroscience intermediate-level care unit model: retrospective analysis of impact on patient flow and safety. Neurohospitalist. 2017;7:83-90.

15. Sekhon M, Gooderham $P$, Toyota B, et al. Implementation of neurocritical care is associated with improved outcomes in traumatic brain injury. Can J Neurol Sci. 2017:44:350-7.

16. Soliman I, Aletreby WT, Faqihi F, et al. Improved outcomes following the establishment of a neurocritical care unit in Saudi Arabia. Crit Care Res and Pract 2018; Article ID 2764907:1-6.

17. Sarpong Y, Nattanmai P, Schelp G, et al. Improvement in quality metrics outcomes and patient and family satisfaction in a neurosciences intensive care unit after creation of a dedicated neurocritical care team. Crit Care Res and Pract 2017; Article ID 6394105;1-6. 
18. McCredie VA, Alali AS, Scales DC, et al. Impact of ICU structure and processes of care on outcomes after severe traumatic brain injury: a multicenter cohort study. Crit Care Med. 2018;46:1139-49.

19. Tepas JJ, Pracht EE, Orban BL, Flint LM. High-volume trauma centers have better outcomes treating traumatic brain injury. J Trauma Acute Care Surg. 2013;74:143-7.

20. Pandey AS, Gemmete JJ, Wilson TJ, et al. High subarachnoid hemorrhage patient volume associated with lower mortality and better outcomes. Neurosurgery. 2015;77:462-70.

21. Quinn T, Moskowitz J, Khan MW, et al. What families need and physicians deliver: contrasting communication preferences between surrogate decision-makers and physicians during outcome prognostication in critically ill TBI patients. Neurocrit Care. 2017;27:154-62.

22. Weber U, Johnson J, Anderson N, et al. Dedicated afternoon rounds for ICU patients' families and family satisfaction with care. Crit Care Med. 2018;46:602-11.

23. Carney N, Totten AM, O'Reilly C, et al. Guidelines for the management of severe traumatic brain injury. New York: Brain Trauma Foundation; 2016.

24. Diringer MN, Bleck TP, Hemphill JC, et al. Critical care management of patients following aneurysmal subarachnoid hemorrhage: recommendations from the Neurocritical Care Society's Multidisciplinary Consensus Conference. Neurocrit Care. 2011;15:211-40.
25. Hemphill JC, Greenberg SM, Anderson CS, et al. Guidelines for the management of spontaneous intracerebral hemorrhage: a guideline for healthcare professionals from the American Heart Association/American Stroke Association. Stroke. 2015;46:2032-60.

26. Shrestha GS, Suarez Jl, Hemphill JC. Precision medicine in neurocritical care. JAMA Neurol. 2018;75:1463-4.

27. Venkatasubba CP, Suarez Jl, Martin RH, et al. Global survey of outcomes of neurocritical care: analysis of the PRINCE Study Part 2.

28. Suarez Jl, Martin RH, Bauza C, et al. Worldwide organization of neurocritical care: results from the PRINCE Study Part 1.

29. Taylor CA, Bell JM, Breiding MJ, Xu L. Traumatic brain injury-related emergency department visits, hospitalizations, and deaths - United States, 2007 and 2013. MMWR Surveill Summ. 2017;66:1-16.

30. Nichol G, Thomas E, Callaway CW, et al. Regional variation in out-ofhospital cardiac arrest incidence and outcome. JAMA. 2008;300:1423-31.

31. Etiman N, Chang HS, Hackenberg K, et al. Worldwide incidence of aneurysmal subarachnoid hemorrhage according to region, time period, blood pressure, and smoking prevalence in the population: a systematic review and meta-analysis. JAMA Neurol. 2019;76:588.

32. Rincon F, Mayer SA. The epidemiology of intracerebral hemorrhage in the United States from 1979 to 2008. Neurocrit Care. 2013;19:95-102. 